\title{
Direct synthesis of a metalloporphyrin complex on a surface
}

\author{
J. Michael Gottfried, Ken Flechtner, Andreas Kretschmann, Thomas Lukasczyk, and Hans-Peter Steinrück
}

\section{Supporting Information}

All experiments were performed with a commercial $\mathrm{X}$-ray photoelectron spectrometer (Scienta ESCA200) equipped with an Al- $\mathrm{K}_{\alpha} \mathrm{X}$-ray source $(1486.6 \mathrm{eV})$, a monochromator, and a hemispherical energy analyzer (SES-200). The overall energy resolution amounts to $0.3 \mathrm{eV}$. The base pressure in this stainless steel ultra-high vacuum (UHV) system is below $1 \cdot 10^{-10}$ mbar. In addition to the XP spectrometer, the system is equipped with a differentially pumped gas discharge lamp, LEED optics (ErLEED-1000A), two ion guns for sample cleaning and ion-scattering experiments, a mass spectrometer (Balzers QMS 112), and several evaporators. For evaporation of the porphyrins, we used a home-built Knudsen cell evaporator, while cobalt was evaporated with a commercial electron beam evaporator (Omicron EFM-3). Fluxes were measured with a quartz crystal microbalance. The porphyrins were evaporated at a temperature of $638 \mathrm{~K}$, measured at the crucible and resulting in a flux of typically $0.05 \AA / \mathrm{s}$, and were adsorbed on a $10 \mathrm{~mm} \times 10 \mathrm{~mm}$ square Ag single crystal (purity > $99.999 \%$ ) with a thickness of $2 \mathrm{~mm}$ and a polished (111) surface with an alignment of $<0.1^{\circ}$. Two type $\mathrm{K}$ thermocouples were mounted directly on the $\mathrm{Ag}$ crystal for precise temperature measurements. The crystal was mounted to a manipulator that allows for cooling and heating in the temperature

\section{Supporting Figures}

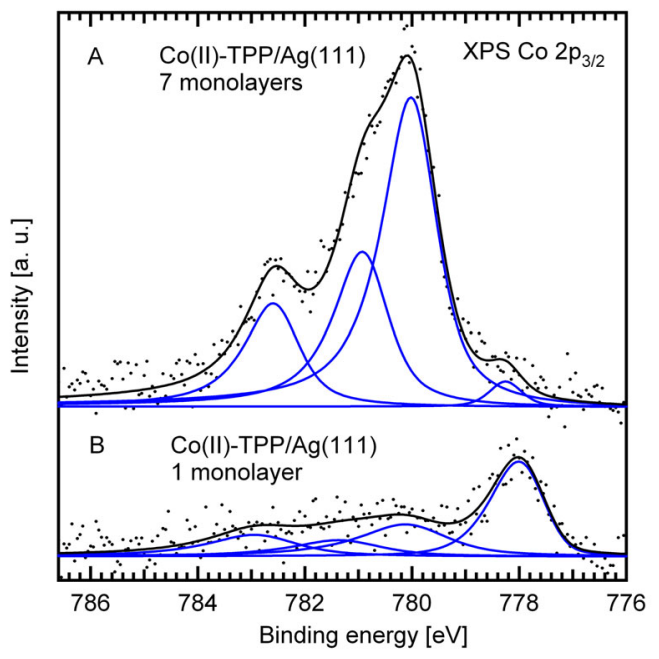

Figure S1: Co $2 \mathrm{p}_{3 / 2}$ XP spectra of Co(II)-TPP. (A) multilayer and (B) monolayer. The splitting of the signals is due to final states of different spin and other final state effects as will be discussed elsewhere. ${ }^{4}$ range $95 \mathrm{~K}$ - $900 \mathrm{~K}$. During the evaporation deposition of the porphyrins, the sample was kept at room temperature and, thereafter, heated to $500 \mathrm{~K}$ in order to desorb excessive porphyrin molecules, i.e., molecules in the second or higher layers. After this annealing step, the LEED patterns of the well ordered monolayers were observed. The UV photoelectron spectra presented in Figure S2 were taken with He-I radiation $(21.22 \mathrm{eV})$ and a sample bias of $-10 \mathrm{~V}$. The overall energy resolution for UPS was $<0.1 \mathrm{eV}$. The Fermi edge of the clean Ag surface was used as a reference for the reported XPS and UPS binding energies $\left(E_{B} \equiv 0\right)$.

The investigated porphyrins, tetraphenylporphyrin and $\mathrm{Co}$ (II)-tetraphenylporphyrin (purity $>98 \%$, Porphyrin Systems GbR), were degassed in vacuo for $24 \mathrm{~h}$ at $150^{\circ} \mathrm{C}$ prior to the evaporation deposition. The Co wire used for evaporation was of the purity $>99.99 \%$ (Goodfellow).

In this manuscript, "monolayer" refers to a saturated layer of molecules that have direct contact to the substrate surface. In contrast, the unit "ML" is defined as the number of adsorbed molecules or atoms per unit area divided by the number of substrate atoms per unit area. For example, the monolayer of $\mathrm{Co}$ (II)-TPP corresponds to $0.043 \mathrm{ML}$.

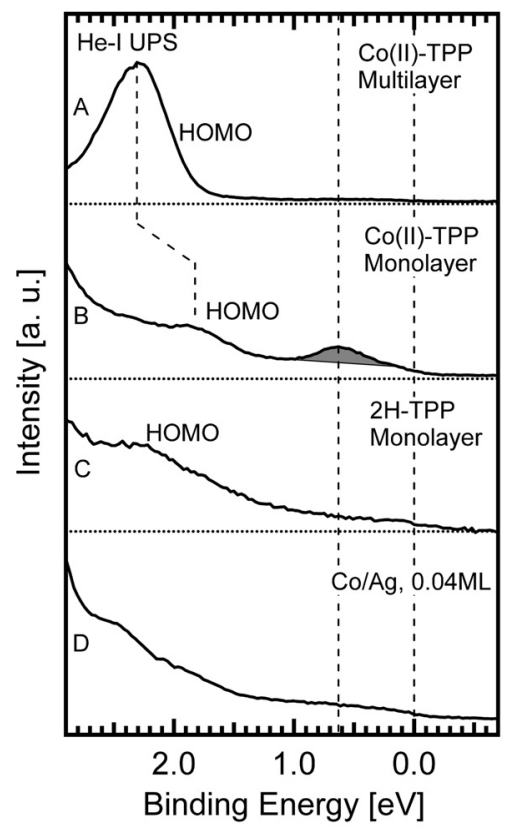

Figure S2: He-I UP spectra $(\mathrm{h} v=21.22 \mathrm{eV})$ : (A) Co(II)-TPP multilayer (4 monolayers), (B) Co(II)-TPP monolayer, (C) 2H-TPP monolayer, (D) Co submonolayer (0.04 ML). The shaded peak at $0.6 \mathrm{eV}$ in Graph B reflects the $\mathrm{Co}(\mathrm{II})-\mathrm{Ag}$ interaction. 